\title{
(6) OPEN ACCESS \\ Reducing musculoskeletal injury and concussion risk in schoolboy rugby players with a pre-activity movement control exercise programme: a cluster randomised controlled trial
}

\author{
Michael D Hislop, ${ }^{1}$ Keith A Stokes, ${ }^{1}$ Sean Williams, ${ }^{1}$ Carly D McKay, ${ }^{1}$ Mike E England, ${ }^{2}$ \\ Simon P T Kemp, ${ }^{2}$ Grant Trewartha'
}

- Additional material are available. To view these files please visit the journal online (http://dx.doi.org/10.1136/ bjsports-2016-097434).

${ }^{1}$ Department for Health, University of Bath, Bath, UK ${ }^{2}$ Rugby Football Union, Twickenham, UK

Correspondence to Keith A Stokes, Department for Health, University of Bath, Bath BA2 7AY, UK; k.stokes@bath. ac.uk

Accepted 12 April 2017

Published Online First

17 May 2017
CrossMark

To cite: Hislop MD, Stokes KA, Williams $S$, et al. Br I Sports Med 2017:51:1140-1146.

\begin{abstract}
Background Injury risk in youth rugby has received much attention, highlighting the importance of establishing evidence-based injury reduction strategies. Aim To determine the efficacy of a movement control exercise programme in reducing injuries in youth rugby players and to investigate the effect of programme dose on injury measures.
\end{abstract}

Methods In a cluster-randomised controlled trial, 40 independent schools (118 teams, 3188 players aged 14-18years) were allocated to receive either the intervention or a reference programme, both of which were to be delivered by school coaches. The intervention comprised balance training, whole-body resistance training, plyometric training, and controlled rehearsal of landing and cutting manoeuvres. Time-loss ( $>24$ hours) injuries arising from school rugby matches were recorded by coaches and medical staff.

Results 441 time-loss match injuries (intervention, 233; control, 208) were reported across 15938 match exposure-hours (intervention, 9083; control, 6855). Intention-to-treat results indicated unclear effects of trial arm on overall match injury incidence (rate ratio $(R R)=0.85,90 \%$ confidence limits 0.61 to 1.17), although clear reductions were evident in the intervention arm for concussion incidence ( $R R=0.71$, 0.48 to 1.05 ). When trial arm comparisons were limited to teams who had completed three or more weekly programme sessions on average, clear reductions in overall match injury incidence $(R R=0.28,0.14$ to 0.51 ) and concussion incidence ( $R R=0.41,0.17$ to 0.99 ) were noted in the intervention group.

Conclusion A preventive movement control exercise programme can reduce match injury outcomes, including concussion, in schoolboy rugby players when compared with a standardised control exercise programme, although to realise the greatest effects players should complete the programme at least three times per week.

\section{INTRODUCTION}

The injury risk in youth rugby has received attention in the mainstream public and sports medicine literature $^{12}$ and has prioritised the formulation of appropriate preventive measures. ${ }^{3}$ Musculoskeletal injuries and concussion are prominent reasons for time loss from sport for adolescent rugby players, ${ }^{4}$ 5 and significant youth sports injuries, in general, have been implicated in long-term disability and compromised quality of later life. ${ }^{67}$ Conditioning the musculoskeletal system to tolerate external forces, through enhancing strength and movement control, has been advocated as means of reducing musculoskeletal injury risk, as indicated by a growing evidence base that supports using multifaceted preventive exercise programmes to reduce musculoskeletal injury risk across male sports such as basketball ${ }^{8}$ and soccer. ${ }^{9-11}$ Moreover, cross-sectional associations between neck strength measures with concussion risk in young athletes invite the possibility that interventions to enhance neck strength may reduce concussion risk. ${ }^{12}$ The injury patterns in rugby differ from other team sports, owing to a greater frequency of concussion, upper body and contact-related injuries, ${ }^{13}{ }^{14}$ and so it is uncertain if introducing a targeted exercise programme can reduce musculoskeletal injuries and/or concussion in youth rugby players.

The efficacy of preventive exercise programmes is dependent on several factors, of which the frequency of programme use (ie, dose) may be one such factor. ${ }^{15}$ Dose-response relationships have typically been identified between programme use and reductions in knee ligament injury incidence in female sportspeople. ${ }^{1617}$ Assessing the effects of programme dose can be useful in reinforcing the outcomes of intervention research and informing subsequent implementation attempts. ${ }^{18}$

The aims of this study were to assess the efficacy of a pre-activity movement control exercise intervention to reduce the incidence and burden of rugby-related injuries in a schoolboy population and to assess the influences of programme dose and compliance on injury outcomes.

\section{METHODS}

\section{Study design and recruitment}

A cluster-randomised controlled trial was conducted across independent school rugby teams over one playing season (August to December 2015). The study design was in accordance with the Consolidated Standards of Reporting Trials statement, ${ }^{19}$ and the trial was registered before recruitment (trial registration number: ISRCTN13422001). Study procedures were approved by the Research Ethics Approval Committee for Health at the University of Bath.

Each school was treated as a cluster, within which under-15 (U15), under-16 (U16) and under-18 (U18) teams were allocated to the same trial arm. 
A priori sample size calculations were undertaken, ${ }^{20}$ incorporating injury data collected from a previous study on injuries in schoolboy rugby players. ${ }^{5}$ To discern a $30 \%$ reduction in match injury incidence at $80 \%$ statistical power, 13 schools per trial arm were required. Seven additional schools were recruited in each trial arm to account for attrition, yielding a target sample of 40 schools. Schools deemed eligible to participate had on-site access to physiotherapists or nurses who would treat all rugby-related injuries. Two hundred and twenty schools were identified and sequentially contacted in randomised groups of 60 through trial invitation letters, emails and direct telephone calls to senior members of the sport and rugby programmes until 40 schools had provided written informed consent from a member of the senior management team to participate in the trial. Schools were randomly allocated to the intervention or control groups on a 1:1 basis. The randomisation process for both recruitment and allocation was completed independently of the research team. Schools were briefed that they would receive an exercise programme to be delivered by the coaches to their U15, U16 and U18 teams, but were blinded to their trial arm allocation. All U15, U16 or U18 players who participated in training or match play at the school were eligible to participate in the trial. Coach consent (in loco parentis), player assent and opportunity for parental opt-out (via school-parent mailing lists) were sought.

Coaches attended a pre-study workshop (typically 1 hour duration) led by the research team at each school (June to July 2015), at which they were introduced to either the intervention or the control exercise programme and data collection materials, in addition to being provided with a practical demonstration of the programme. The practical demonstration of the programme entailed coaches observing a research team member leading a group of youth rugby players (U15, U16 or U18) through a session. Coaches across both trial arms received identically formatted data collection and programme materials (except for exercises). Programme materials included a filmed demonstration of the exercises (as a DVD), laminated cue cards (images and key coaching cues for each exercise) and a booklet detailing how to complete individual exercises. A further meeting was arranged at all schools during pre-season for members of the research team to collect informed assent and baseline anthropometric information (standing height, seated height and body mass) from all players involved in the trial.

\section{The exercise programmes being trialled}

The process of devising the intervention and control exercise programmes have been reported elsewhere. ${ }^{21}$ Both programmes comprised four phases, with progressions occurring via increased difficulty and repetition volume of exercises. Phase 1 was devised for the pre-season period (typically 1-2 weeks' duration). Both programmes progressed to phase 2 on the school term commencing in September 2015, with two further planned phase progressions occurring every 4 weeks until the end of the study in December. This timing enabled players to master the exercises before being introduced to a more advanced phase. Progression of the exercises was undertaken at the team level, with all players within the same age group teams completing the same exercises at the same time. Phase difficulty was offset by age group (ie, phase 3 of the U15 programme was similar in difficulty to phases 2 and 1 of the U16 and U18 programmes, respectively) to maintain a sufficient stimulus for players at an appropriate level. Both programmes were intended to take place during the first $20 \mathrm{~min}$ of each pitch-based training session and match warm-up, and coaches in charge of each team acted as delivery agents. Coaches were instructed to use the programme materials, particularly the laminated cue cards during sessions, to assess movement execution in relation to the specified cues for each exercise and to identify movements that could be improved.

The exercise programme allocated to teams in the intervention group integrated balance/perturbation training, resistance training, plyometric training and controlled rehearsal of sport-specific landing and cutting manoeuvres with verbal feedback and reinforcement of technique from the coach (see online supplementary material). The exercise programme allocated to the control cohort was derived from currently regarded best practice within schoolboy rugby and featured a running-based warm-up, dynamic stretching, controlled wrestling, mobility and speed/ change of direction-related drills (without the specific feedback instructions given in the intervention programme). The content within both the intervention and control programmes were categorised into four separate parts (parts A, B, C and D) to aid the structure of sessions and compliance reporting. A sample phase from the intervention exercise programme has been supplied for supplementary reference.

\section{Data collection}

The definitions of a reportable injury (incurring a time loss of more than 24 hours), match exposure and training exposure (only pitch-based activities) were adapted from the consensus statement for injury definitions and data collection procedures in rugby union. ${ }^{22}$ Coaches recorded training exposure (length of each training session in minutes, number of players attending training session), match exposure (match date, opponent name, list of players selected to play in each fixture) and programme compliance at each session (completion of programme parts A, B, C and D) on weekly paper-based or electronic report forms. Programme compliance indicated the proportion of programme parts that were completed at the team level across all exposures. Coaches started the injury reports by logging the date that the injury occurred, the injured player's trial ID and playing position, and the event leading directly to the injury. School medical staff recorded the injury location and a diagnosis when players visited for treatment. Logging the injury location and diagnosis aligned with the first two levels of the Orchard Sports Injury Classification System (V.10). ${ }^{23}$ Coaches recorded the return-to-play date (date of full participation in training or match play). A member of the research team visited schools periodically (2-3 weeks' interval) during the study period to retrieve completed report forms and to discuss study progress, but not to promote compliance or fidelity with using the programmes.

\section{Statistical analyses}

Statistical analyses were undertaken with IBM SPSS Statistics (V.22.0 for Windows). Trial arm comparisons across baseline player characteristics (age, anthropometric characteristics and maturity timing) were assessed using linear and logistic regression. The odds ratio (OR) generated from the logistic regression was converted to a proportion ratio to permit analysis via magnitude-based inference. ${ }^{24}$

Intention-to-treat analyses compared injury measures between the trial arms for all teams that provided injury and exposure data, regardless of returning complete programme compliance or dose data. Trial arm comparisons were also undertaken on a per-protocol basis with teams that maintained a mean programme completion rate of three or more weekly sessions, which represented a threshold for optimal compliance based on previous 


\section{Original article}

findings. ${ }^{25}$ The effects of intervention-only programme dose on injury were assessed by comparing injury measures between teams that maintained an average of three or more sessions per week with those who averaged completing the programmes less than three sessions per week. Overall match injury incidence (injuries/1000 player-hours) and burden (days lost/1000 player-hours) rates acted as dependent variables, with further stratification by injury location and event. All estimations were made using generalised linear modelling with a Poisson distribution, a log-linear link function and offset for hours of exposure to generate rate ratios (RR) and 90\% confidence limits (90\% CL) for injury incidence and burden values. RR and 90\% CL were assessed against predetermined smallest worthwhile effects in injury outcome. ${ }^{26}$ The smallest worthwhile effects favouring the intervention and favouring the control were given as $\mathrm{RR}=0.90$ and $\mathrm{RR}=1.11$, respectively. ${ }^{27}$ Effects were deemed clear if the per cent likelihood that the true effect favoured the intervention (RR below 0.90) was greater than $25 \%$, and the OR between favouring the intervention and favouring the control was greater than 66 (ie, if the likelihood of effect favouring the intervention was $25 \%$ and the likelihood of effect favouring the control was less than $0.5 \%$ ); otherwise, effects were deemed unclear. Effects were qualified against probabilistic terms from the following scale: $<0.5 \%$, most unlikely; $0.5 \%-5 \%$, very unlikely; 5\%-25\%, unlikely; 25\%-75\%, possibly; 75\%-95\%, likely; 95\%-99.5\%, very likely; >99.5\%, most likely. ${ }^{28}$

\section{RESULTS}

From a target population of 220 potentially eligible schools that were contacted for recruitment, 40 schools (118 teams, 3188 players aged $14-18$ years) consented to participate in the trial and were randomly allocated to the intervention or control group (figure 1). Nine schools and 35 teams dropped out of the
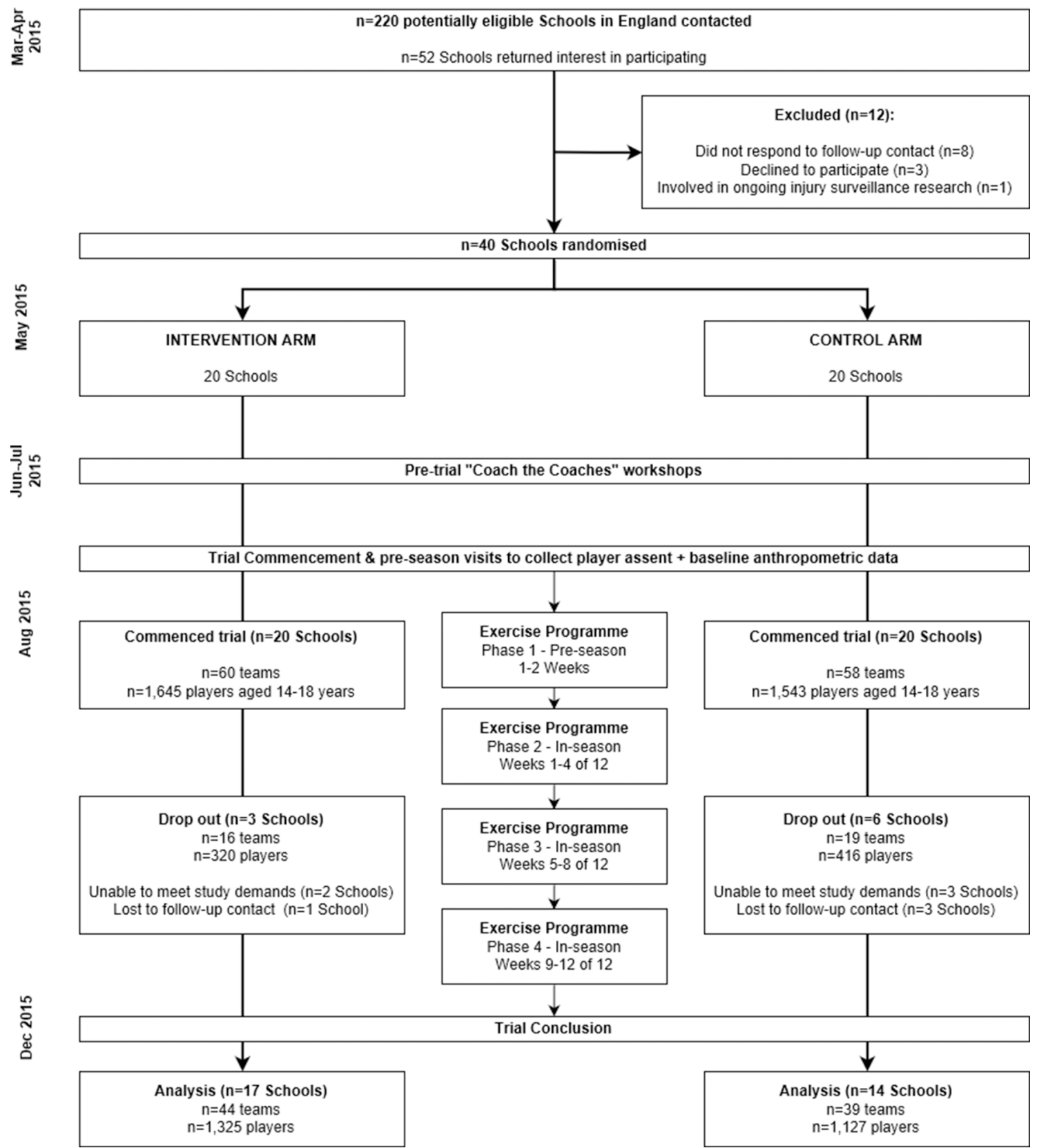

Figure 1 Flow diagram presenting the recruitment and retention of participants through the study. 
Table 1 Summary and comparisons of player characteristics between the intervention ( $n=1325$ players) and control ( $n=1127$ players) groups

\begin{tabular}{|c|c|c|c|c|c|}
\hline Trial arm & Intervention & Control & $\begin{array}{l}\text { Effect size * } \\
(90 \% \mathrm{CL})\end{array}$ & (\% higher | trivial | lower)† Inference & $\mathrm{p}$ Value \\
\hline Age (years) & $16.0 \pm 1.2$ & $15.9 \pm 1.1$ & $0.06(0.00$ to 0.14$)$ & (0|100|0\%) Most likely trivial & 0.19 \\
\hline Stature $(\mathrm{cm})$ & $177.4 \pm 7.3$ & $176.6 \pm 7.5$ & $0.11(0.03$ to 0.19$)$ & (4|96|0\%) Very likely trivial & 0.03 \\
\hline Body mass $(\mathrm{kg})$ & $74.7 \pm 12.9$ & $72.5 \pm 13.1$ & $0.17(0.09$ to 0.25$)$ & (27|73|0\%) Possibly trivial & $<0.01$ \\
\hline \multicolumn{6}{|l|}{ Maturity offset (\%) } \\
\hline$>1$ year post-PHV & $642(71 \%)$ & $586(70 \%)$ & $1.03(0.91$ to 1.16$)$ & (4|95|1\%) Very likely trivial & 0.68 \\
\hline$\leq 1$ year post-PHV & $261(29 \%)$ & $251(30 \%)$ & & & \\
\hline
\end{tabular}

Data presented as mean \pm SD or as raw frequency (\%) where specified.

*Effect sizes for age, stature and body mass expressed as Cohen's d; effect size for maturity offset expressed as a proportion ratio. ${ }^{24}$

†Percentage likelihood of effect being higher or lower is analogous to effect favouring intervention or control, respectively.

$\mathrm{CL}$, confidence limits; PHV, peak height velocity.

trial, with data collected from 31 schools, 83 teams and 2452 players used for analysis (intervention, 17 schools, 44 teams, 1325 players; control, 14 schools, 39 teams, 1127 players). Comparisons of baseline player anthropometric characteristics between the trial arms are outlined in table 1. Trivial differences were detected between the trial arms for mean player age, stature, body mass or the distribution of players by maturity timing (table 1).

\section{Exposure, injury and compliance}

The intervention cohort ( $n=17$ schools, 44 teams) accrued 37346 exposure-hours (match, 9083; training, 28 263), with the control cohort ( $\mathrm{n}=14$ schools, 39 teams) reporting 32375 exposure-hours (match, 6855; training, 25 520). The intervention cohort recorded 233 match injuries (totalling 6499 days lost) and 58 training injuries (1028 days lost), with the control cohort recording 208 match injuries (5907 days lost) and 54 training injuries (1150 days lost). Overall match and training injury incidence in the intervention cohort was $26 / 1000$ hours (23-29) and $2 / 1000$ hours (2-3), and in the control cohort was $30 / 1000$ hours (27-34) and 2/1000 hours (2-3), respectively.

Summary injury and exposure results between the trial arms are outlined in table 2 . Of 441 reported match injuries, 168 were sustained to the head/neck (intervention, 82; control, 86), 125 to the upper limb (intervention, 65; control, 60), 113 to the lower limb (intervention, 67; control, 46) and 35 to the trunk (intervention, 19; control, 16). Of 168 head/neck injuries, 105 were reported as concussion (intervention, 51; control, 54). Match concussion incidence in the intervention group was $6 / 1000$ hours (4-7) and in the control group was 8 (6-10).

Complete compliance data were retrieved from 63 out of 83 teams (intervention, 32 teams; control, 31 teams). In teams who had provided complete compliance information, mean programme completion rate across both trial arms was close

Table 2 Descriptive statistics for match and training injuries across the control and intervention cohorts

\begin{tabular}{|c|c|c|c|c|c|}
\hline & & Intervention ( $n=17$ schools, 44 teams) & Control ( $n=14$ schools, 39 teams) & Rate ratio $(90 \% \mathrm{CL})$ & p Value \\
\hline \multirow[t]{2}{*}{ Exposure-hours } & Match & 9083 & 6855 & - & - \\
\hline & Training & 28263 & 25520 & - & - \\
\hline \multirow[t]{2}{*}{ Injuries } & Match & 233 & 208 & - & - \\
\hline & Training & 58 & 54 & - & - \\
\hline \multirow[t]{2}{*}{ Days lost to injury } & Match & 6499 & 5907 & - & - \\
\hline & Training & 1028 & 1150 & - & - \\
\hline \multirow[t]{2}{*}{ Overall match } & Incidence & $26(23-29)$ & $30(27-34)$ & $0.85(0.61-1.17)$ & 0.40 \\
\hline & Burden & $715(701-730)$ & $862(844-880)$ & $0.83(0.58-1.18)$ & 0.38 \\
\hline \multirow[t]{2}{*}{ Overall training } & Incidence & $2(2-3)$ & $2(2-3)$ & $0.97(0.52-1.81)$ & 0.94 \\
\hline & Burden & $36(34-38)$ & $45(43-48)$ & $0.80(0.40-1.60)$ & 0.60 \\
\hline \multicolumn{6}{|c|}{ Match injury by location } \\
\hline \multirow[t]{2}{*}{ Head/neck } & Incidence & $9(7-11)$ & $13(10-15)$ & $0.72(0.51-1.01)$ & 0.11 \\
\hline & Burden & $260(252-269)$ & $285(274-296)$ & $0.91(0.55-1.51)$ & 0.77 \\
\hline \multirow[t]{2}{*}{ Upper limb } & Incidence & $7(6-9)$ & $9(7-11)$ & $0.82(0.51-1.31)$ & 0.48 \\
\hline & Burden & $229(221-238)$ & $345(333-356)$ & $0.66(0.40-1.10)$ & 0.18 \\
\hline \multirow[t]{2}{*}{ Trunk } & Incidence & $2(1-3)$ & $2(1-3)$ & $0.90(0.47-1.71)$ & 0.78 \\
\hline & Burden & $36(32-39)$ & $43(38-47)$ & $0.84(0.35-2.01)$ & 0.74 \\
\hline \multirow[t]{2}{*}{ Lower limb } & Incidence & $7(6-9)$ & $7(5-8)$ & $1.10(0.7-1.72)$ & 0.73 \\
\hline & Burden & $190(182-197)$ & $189(181-198)$ & $1.00(0.52-1.93)$ & 1.00 \\
\hline \multicolumn{6}{|c|}{ Match injury by event } \\
\hline \multirow[t]{2}{*}{ Contact } & Incidence & $22(20-25)$ & $27(23-30)$ & $0.85(0.60-1.19)$ & 0.42 \\
\hline & Burden & $607(594-621)$ & $689(673-706)$ & $0.88(0.60-1.29)$ & 0.59 \\
\hline \multirow[t]{2}{*}{ Non-contact } & Incidence & $2(1-3)$ & $2(1-3)$ & $0.94(0.50-1.77)$ & 0.88 \\
\hline & Burden & $77(72-81)$ & $121(114-128)$ & $0.63(0.25-1.64)$ & 0.43 \\
\hline
\end{tabular}

Incidence values presented as injuries/1000 hours. Burden values presented as days lost/1000 hours.

$\mathrm{CL}$, confidence limits. 


\section{Original article}

to twice per week (intervention, 1.9 sessions/week; control, 2.0 sessions/week). Twelve out of 63 teams maintained a mean weekly programme completion rate of three or more sessions (intervention, seven teams; control, five teams). Compliance to the exercise programmes (proportion of programme parts that were completed) were $69 \%$ and $83 \%$ across the intervention and control arms, respectively.

\section{Intention-to-treat analyses}

Intention-to-treat analyses on the effect of trial arm (intervention, 17 schools, 44 teams; control, 14 schools, 39 teams) were unclear for overall match injuries (incidence $\mathrm{RR}=0.85,90 \% \mathrm{CL}$ : 0.61 to 1.17 ; burden $\mathrm{RR}=0.83,0.58$ to 1.18 ) (figure 2). Effects were also unclear for contact-related injuries (incidence $R R=0.85$, 0.60 to 1.19 ; burden $\mathrm{RR}=0.88,0.60$ to 1.29 ). However, clear effects favouring the intervention programme were noted for head/neck injuries (incidence $\mathrm{RR}=0.72,0.51$ to 1.01 ), upper limb injuries (burden $\mathrm{RR}=0.66,0.40$ to 1.10 ) and concussion (incidence $\mathrm{RR}=0.71,0.48$ to 1.05 ).

\section{Per-protocol analyses}

Per-protocol trial arm comparisons (intervention, four schools, seven teams; control, three schools, five teams) revealed that teams completing the intervention programme three times or more per week suffered $72 \%$ fewer overall match injuries (incidence $\mathrm{RR}=0.28,0.14$ to 0.51 ), $72 \%$ fewer contact-related

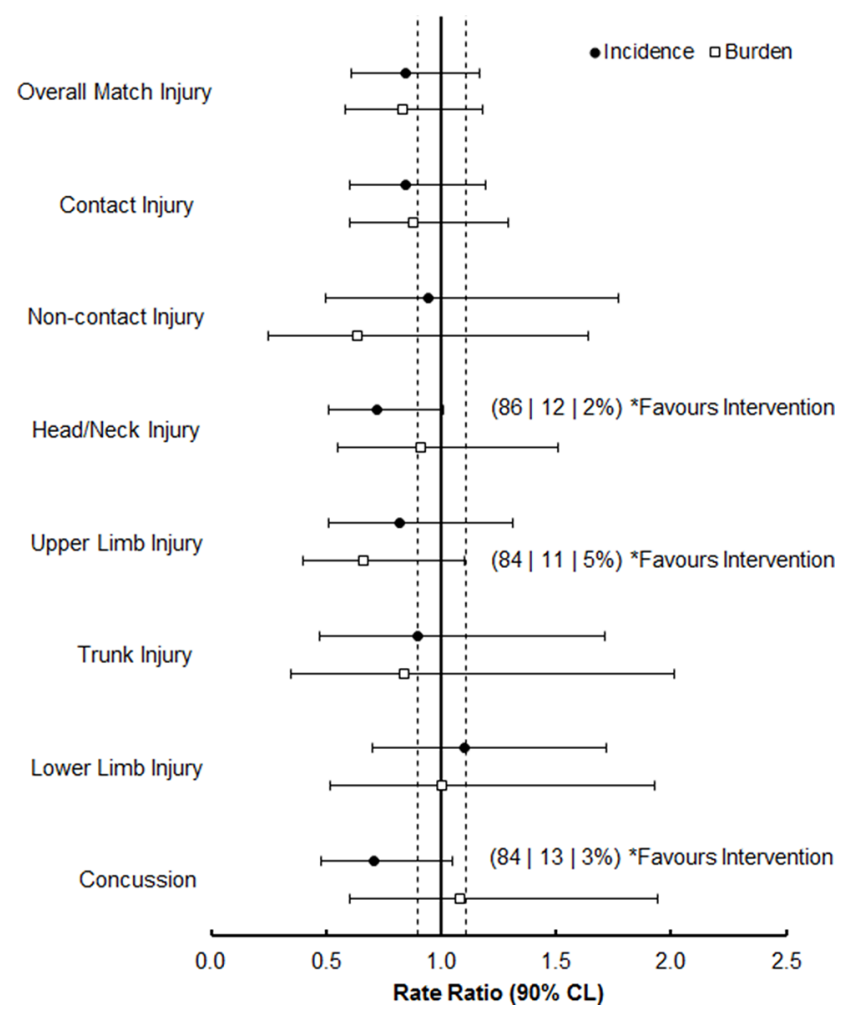

Figure 2 Forest plot detailing the results of the intention-totreat analyses for the effects of trial arm on injury measures ( $n=31$ schools, 83 teams). Data points represent RR of injury measures in the intervention arm relative to the control arm (reference group, $R R=1.00$ ). Dotted vertical lines represent thresholds for smallest worthwhile effects ( $R R=0.90$ and 1.11). Data labels represent the per cent likelihood that each effect favours the intervention | is trivial | favours the control, for outcome variables that demonstrate a clear effect of trial arm allocation. * Likely, ${ }^{* *}$ very likely, ${ }^{* *}$ most likely.

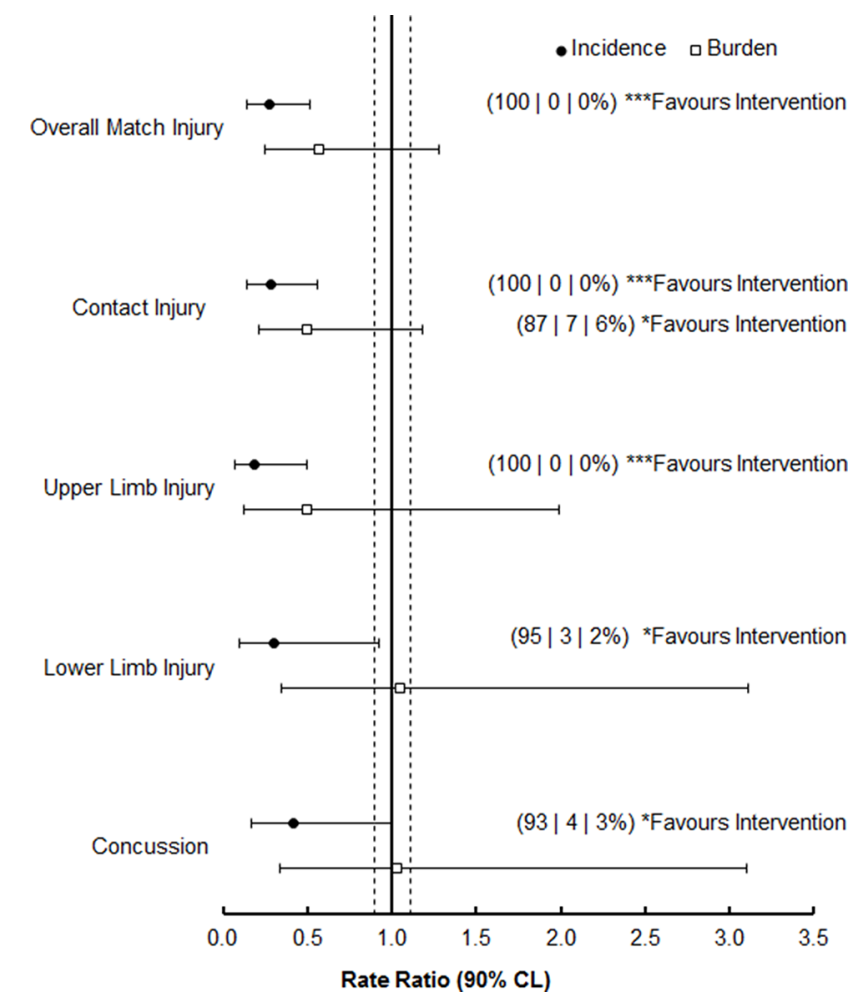

Figure 3 Forest plot illustrating the results of the per-protocol analyses on the effect of trial arm on injury measures in teams with a mean programme completion rate of more than three completions per week ( $n=7$ schools, 12 teams). Data points represent RR of injury measures in the intervention arm relative to the control arm (reference group, $R R=1.00$ ). Dotted vertical lines represent thresholds for smallest worthwhile effects ( $R R=0.90$ and 1.11). Data labels represent the per cent likelihood that each effect favours the intervention | is trivial | favours the control, for outcome variables which demonstrate a clear effect of trial arm allocation. * Likely, ${ }^{* * *}$ most likely. CL, confidence limits.

injuries (incidence $\mathrm{RR}=0.28,0.14$ to 0.56 ), $50 \%$ fewer days lost to contact injuries (burden $\mathrm{RR}=0.50,0.21$ to 1.18 ), $81 \%$ fewer upper limb injuries (incidence $\mathrm{RR}=0.19,0.07$ to 0.50 ), $70 \%$ fewer lower limb injuries (incidence $R R=0.30,0.10$ to 0.92 ) and $59 \%$ fewer concussions (incidence $R R=0.41,0.17$ to 0.99 ) than teams that completed the control programme three or more times per week (figure 3).

Per-protocol analyses conducted in the intervention arm indicated that teams typically completing at least three sessions per week with the intervention programme (four schools, seven teams) suffered $39 \%$ fewer match injuries (incidence $R R=0.61$, 0.42 to 0.88 ), $48 \%$ fewer days lost to match injuries (burden $\mathrm{RR}=0.52,0.29$ to 0.93 ), $42 \%$ fewer match contact injuries (incidence $\mathrm{RR}=0.58,0.41$ to 0.82 ) and $55 \%$ fewer days lost to match contact injuries (burden $\mathrm{RR}=0.45,0.25$ to 0.82 ) than teams that completed the intervention programme fewer than three times per week (10 schools, 25 teams). Effects of intervention programme dose were unclear for upper limb injuries, lower limb injuries and concussion.

\section{DISCUSSION}

This study aimed to determine the efficacy of a movement control exercise programme for preventing injury in youth rugby players and to assess the effect of programme dose on injury measures. Following intention-to-treat analyses, effects 


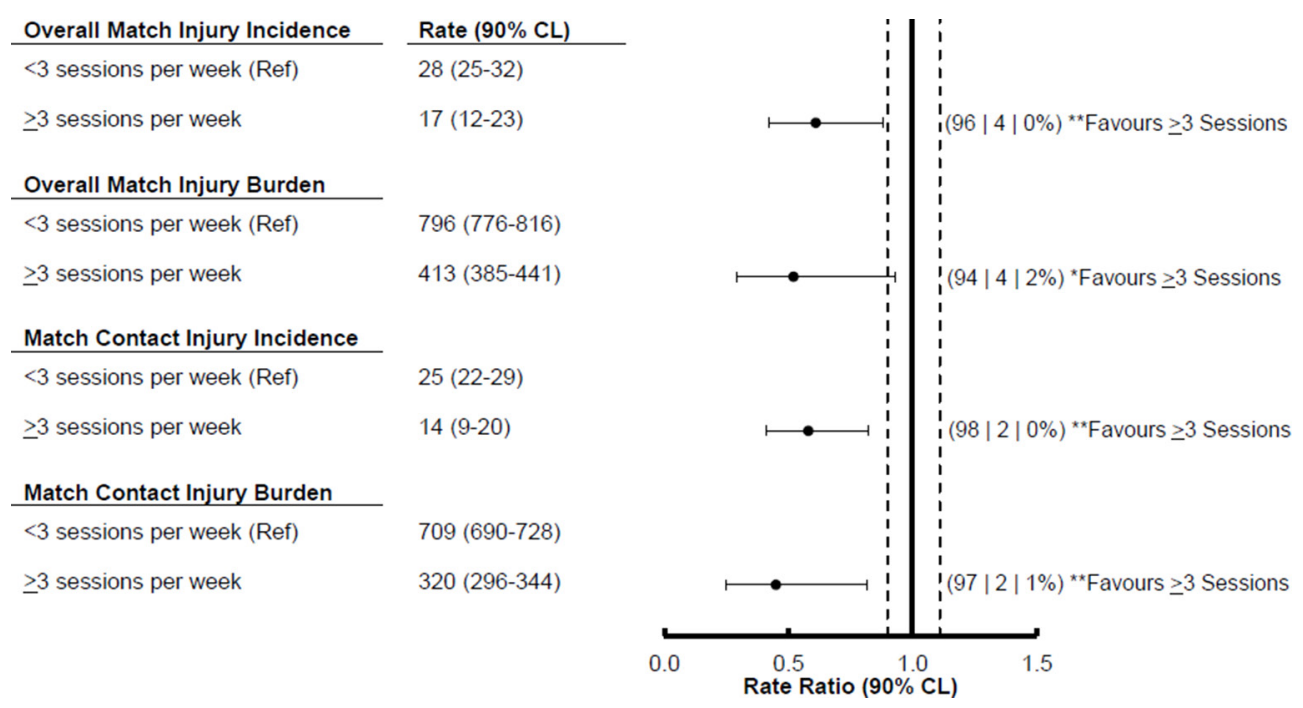

Figure 4 Forest plot illustrating the effects of intervention programme dose on injury measures ( $\mathrm{n}=14$ schools, 32 teams). Data points represent $R R$ of injury measures in teams completing $>3$ sessions per week relative to teams completing $<3$ sessions per week $(R R=1.00)$. Dotted vertical lines represent thresholds for smallest worthwhile effect $(R R=0.90$ and 1.11). Data labels represent the per cent likelihood that each effect favours $>3$ completions per week | is trivial | favours $<3$ completions per week, for outcome variables which demonstrate a clear effect of programme dose. *Likely, ** very likely. CL, confidence limits.

of the trial arm were unclear for overall match injury. Per-protocol trial arm comparisons under conditions of high dose ( $\geq 3$ weekly programme sessions) revealed clear reductions for the intervention arm for overall match injury, contact-related injury, upper and lower extremity injury and concussion incidence values. Moreover, a higher intervention programme dose was shown to reduce the incidence and burden of overall match injuries and contact injuries compared with lower intervention programme doses ( $<3$ weekly programme completions).

\section{Intention-to-treat analyses}

Intention-to-treat analyses indicated unclear effects of trial arm for overall match injury (incidence $R R=0.85$, burden $\mathrm{RR}=0.83$ ) and match contact injury (incidence $\mathrm{RR}=0.85$; burden $\mathrm{RR}=0.88$ ). The $15 \%$ reduction in overall match injury incidence in this study is lower than the $41 \%-56 \%$ reductions noted in other studies conducted in male basketball and soccer players, ${ }^{8} 1011$ which may be partly attributed to differences between definitions of reportable injuries, programme content, or the distribution of injury types and locations between the respective sports, that is, proportion of non-contact lower limb injuries. However, clear effects favouring the intervention exercise programme were noted for head/neck injury incidence (incidence $\mathrm{RR}=0.72$ ) and concussion incidence (incidence $\mathrm{RR}=0.71$ ). Sixty-two per cent of reported head/neck injuries in the trial were attributed to concussion, and therefore the reductions in head/neck injury incidence were likely because of reductions in concussion incidence. Concussion is a priority for prevention across contact and collision sports due to potential concern over medium-term and long-term player welfare. ${ }^{29} 30$ Thus, the substantially reduced concussion incidence across the intervention arm is a very promising finding with regards to current efforts to reduce the risk of concussion.

Neck strength has been shown to be substantially lower in adolescent rugby players when compared with adult players despite similar peripheral strength profiles. ${ }^{31}$ Increased concussion risk is associated with lower neck strength, highlighting this characteristic as a potentially modifiable risk factor. ${ }^{12}$ Enhancing neck muscle strength may prevent concussion by improving the dissipation of impact forces transmitted to the brain. ${ }^{32} 33$ Therefore, it is possible that the neck resistance exercises in the intervention exercise programme contributed to reduced concussion incidence via this mechanism. Neck pain is a common physical complaint among young sportspeople participating in collision sports ${ }^{3435}$ and may be associated with increased concussion risk. ${ }^{33}$ Given that acute and cumulative rugby exposure can adversely impact neck function, ${ }^{36-38}$ the neck resistance exercises may also have contributed to preserving neck function during the playing season, ${ }^{39}$ in turn reducing concussion incidence.

Upper limb injuries are common in contact sports and can also result in substantial time loss in youth rugby players. ${ }^{50}$ Teams in the intervention trial arm suffered substantially fewer days absence due to upper limb injuries than teams in the control arm (burden $\mathrm{RR}=0.66$ ). Little is known about the underlying risk factors and mechanisms for upper limb injuries, and examples of evidence-based upper limb injury prevention are scarce. ${ }^{41}$ Reduced glenohumeral rotation and rotator cuff muscle strength imbalances may be modifiable risk factors for shoulder injuries in rugby players. ${ }^{42}$ The intervention programme may have improved joint kinematics and force-handling capabilities within the upper limb as a result of incorporating resistance and plyometric training of upper body regions, ${ }^{43}$ thus implying that reducing upper limb injury risk across youth contact sports is possible through improving upper limb strength, stability and mobility.

\section{Per-protocol analyses}

The lack of clear substantial effects for overall match and contact-related injuries following intention-to-treat analyses should be considered in the context of dose, which may have affected these outcomes. Although mean intervention programme dose in this study was higher (1.9 completions per week) than has been reported in previous studies (1.3-1.4 completions per week), ${ }^{45}$ the level of dose that teams received in the intervention trial arm may have been insufficient to demonstrate a clear effect on overall match injuries. Greater effects of preventive exercise programmes may be realised if regularly used at least three times per week. ${ }^{25}$ Trial arm comparisons conducted 
on a per-protocol basis showed that intervention trial arm teams that regularly completed the programme more than three times per week suffered $72 \%$ fewer overall match injuries, $72 \%$ fewer contact-related injuries, 81\% fewer upper limb injuries, $70 \%$ fewer lower limb injuries and 59\% fewer concussions than control teams with an equivalent dose. When per-protocol analyses were conducted within the intervention arm, teams that regularly completed the programme over three times per week suffered 39\% fewer match injuries, $48 \%$ fewer days lost to match injuries, $42 \%$ fewer contact injuries and 55\% fewer days lost to contact injuries than teams with less than three weekly programme completions. Regularly performing a preventive exercise programme three times per week over a sustained period has been shown to improve markers for neuromuscular control and muscle strength in male soccer players. ${ }^{47}$ Therefore, these physiological benefits may explain the enhanced effects noted in this study with a high dose of the intervention programme use compared with the control programme and a lower dose of the intervention programme.

Evidence of a dose-response effect on overall and contact-related injuries in this study presents wider applications of the dose-response effect of preventive exercise programmes, and has the potential to inform subsequent implementation attempts through identifying a minimum effective dose in this population. The collective findings from the intention-to-treat and per-protocol analyses highlight that teams involved in contact sports can obtain benefit from using preventive exercise programmes, but more importantly, regular exposure of more than three times per week can result in substantial injury risk reduction.

\section{Limitations and future directions}

There were several limitations to this study that should be acknowledged. First, the research team members that ran the pre-trial workshops and conducted pre-season visits were not blinded to the programme allocation for each school, creating potential bias between the two groups in terms of the processes followed and information disseminated at these workshops; this was mitigated through use of a unified workshop format. Second, individual player compliance was not monitored during the study. Results of previous studies have indicated that individual player compliance may be a more sensitive measure than team compliance in determining the influence of compliance on programme efficacy, ${ }^{4546}$ but puts considerable strain on coaches, and so was not feasible in this setting. Third, it was not possible to validate coach compliance reports or to monitor exercise fidelity (the quality of performing the exercises) through unannounced visits or observations, given that schools have strict policies around access to premises. The fidelity with which teams used the programmes is uncertain, but may actually have mediated programme efficacy along with dose and compliance. ${ }^{48}$

Further work is required to understand the mechanistic bases by which the intervention exercise programme reduced injury outcomes, particularly in relation to the proposed effects of the programme on neck strength and function in reducing concussion incidence, as well as kinematics and force handling capacities in the upper limb. Determining efficacy is a crucial step towards effecting a public health impact of injury prevention measures in rugby, although results of this controlled trial alone are not sufficient to translate to reducing injuries in 'real world' contexts. $^{4950}$ Further research is required to further understand the contexts into which the exercise programme would be implemented, as well as identifying what factors may facilitate or inhibit programme use. ${ }^{18}$ In particular, studies should be directed to identifying what factors could facilitate or inhibit teams from completing the intervention programme three times per week.

\section{CONCLUSION}

Although intention-to-treat results highlighted trivial effects of the intervention exercise programme on overall match injury incidence when compared with the control exercise programme, a substantial reduction in overall match injury incidence was evident from per-protocol trial arm comparisons under conditions of high programme dose (three or more weekly sessions). Notable beneficial effects of the preventive programme on upper and lower extremity injuries and concussion incidence also have important implications for the reduction of these priority injury types in youth rugby.

\section{What are the findings?}

Intention-to-treat analyses revealed that the intervention programme substantially reduced upper limb injury burden ( $R R=0.66,90 \%$ CL 0.40 to 1.10 ) and concussion incidence ( $R R=0.71,90 \%$ CL 0.48 to 1.05 ) compared with the control programme.

- Completing the intervention programme 3 times per week led to substantial reductions of $72 \%$ in overall match injury incidence ( $R R=0.28,90 \% \mathrm{CL} 0.14$ to 0.51 ) and $72 \%$ in contact-related injury incidence $(\mathrm{RR}=0.28,90 \% \mathrm{CL}$ 0.28 to 0.56 ) compared with the control programme.

\section{How might it impact on clinical practice in the future?}

- These findings provide encouraging evidence that a pre-activity preventive exercise programme can substantially reduce injury risk in youth rugby.

- Notable reductions in upper extremity injuries and concussion offer promising implications for the prevention of these high-risk injury types in young rugby players.

- Findings also outline a minimum effective dose of three sessions a week, whereby programme efficacy can be optimised.

Acknowledgements The authors would like to acknowledge with sincere gratitude all school coaches and medical staff for the recording of injury, exposure and compliance data during the study period. Furthermore, thanks are expressed to members of the project's 'Technical Project Group' who contributed to the development of both exercise programmes: Matthew Cross, Alasdair Dempsey, Carolyn Emery, Richard Mack, Katie Morris, Simon Roberts, Des Ryan, Evert Verhagen, Sean Williams, Shaun Williams.

Contributors GT, KAS, MEE and SK initiated the overall project. MDH, KAS, SW CDM, MEE, SK and GT conceived and designed the study. MDH analysed the data and prepared the first draft of the manuscript. All authors made substantial contributions to revision of the manuscript prior to submission.

\section{Competing interests None declared.}

Ethics approval Research Ethics Approval Committee for Health (REACH) at the University of Bath.

\section{Provenance and peer review Not commissioned; externally peer reviewed.}

Open Access This is an Open Access article distributed in accordance with the Creative Commons Attribution Non Commercial (CC BY-NC 4.0) license, which permits others to distribute, remix, adapt, build upon this work non-commercially, and license their derivative works on different terms, provided the original work is properly cited and the use is non-commercial. See: http://creativecommons.org/ licenses/by-nc/4.0/

(c) Article author(s) (or their employer(s) unless otherwise stated in the text of the article) 2017. All rights reserved. No commercial use is permitted unless otherwise expressly granted. 


\section{REFERENCES}

1 Carter M. The unknown risks of youth rugby. BMJ 2015;350:h26

2 Pollock AM, Kirkwood G. Removing contact from school rugby will not turn children into couch potatoes. Br J Sports Med 2016;50:963-4.

3 Tucker R, Raftery M, Verhagen E. Injury risk and a tackle ban in youth Rugby Union: reviewing the evidence and searching for targeted, effective interventions. A critical review. Br J Sports Med 2016:50:921-5.

4 Archbold HA, Rankin AT, Webb M, et al. RISUS study: rugby injury surveillance in Ulster schools. Br J Sports Med 2017.51.

5 Palmer-Green DS, Stokes KA, Fuller CW, et al. Match injuries in English youth academy and schools rugby union: an epidemiological study. Am J Sports Med 2013:41:749-55.

6 Maffulli N, Longo UG, Gougoulias N, et al. Long-term health outcomes of youth sports injuries. Br I Sports Med 2010;44:21-5.

7 Maffulli N, Longo UG, Spiezia F, et al. Sports injuries in young athletes: long-term outcome and prevention strategies. Phys Sportsmed 2010;38:29-34.

8 Longo UG, Loppini M, Berton A, et al. The FIFA 11+ program is effective in preventing injuries in elite male basketball players: a cluster randomized controlled trial. $\mathrm{Am} J$ Sports Med 2012:40:996-1005.

9 Grooms DR, Palmer T, Onate JA, et al. Soccer-specific warm-up and lower extremity injury rates in collegiate male soccer players. J Athl Train 2013;48:782-9.

10 Owoeye OB, Akinbo SR, Tella BA, et al. Efficacy of the FIFA 11+ warm-up programme in male youth football: a cluster randomised controlled trial. I Sports Sci Med 2014;13:321-8.

11 Silvers-Granelli H, Mandelbaum B, Adeniji 0, et al. Efficacy of the FIFA 11+ injury prevention program in the collegiate male soccer player. Am J Sports Med 2015;43:2628-37.

12 Collins $\mathrm{CL}$, Fletcher EN, Fields SK, et al. Neck strength: a protective factor reducing risk for concussion in high school sports. J Prim Prev 2014;35:309-19.

13 Brooks JH, Kemp SP. Recent trends in rugby union injuries. Clin Sports Med 2008;27:51-73.

14 Junge A, Cheung K, Edwards T, et al. Injuries in youth amateur soccer and rugby players - comparison of incidence and characteristics. Br J Sports Med 2004;38:168-72.

15 Sugimoto D, Myer GD, Micheli LJ, et al. ABCs of evidence-based anterior cruciate ligament injury prevention strategies in female athletes. Curr Phys Med Rehabil Rep 2015;3:43-9.

16 Sugimoto D, Myer GD, Foss KD, et al. Dosage effects of neuromuscular training intervention to reduce anterior cruciate ligament injuries in female athletes: metaand sub-group analyses. Sports Med 2014;44:551-62.

17 Sugimoto D, Myer GD, Barber Foss KD, et al. Critical components of neuromuscular training to reduce $\mathrm{ACL}$ injury risk in female athletes: meta-regression analysis. $\mathrm{Br} J$ Sports Med 2016:50:1259-66.

18 Finch C. A new framework for research leading to sports injury prevention. J Sci Med Sportdiscussion 2006:9:3-9.

19 Schulz KF. CONSORT 2010 statement: updated guidelines for reporting parallel group randomized trials. Ann Intern Med 2010;152:726-32.

20 Hayes RJ, Bennett $\mathrm{S}$. Simple sample size calculation for cluster-randomized trials. Int $J$ Epidemiol 1999;28:319-26.

21 Hislop MD, Stokes KA, Williams S, et al. The efficacy of a movement control exercise programme to reduce injuries in youth rugby: a cluster randomised controlled trial. BMJ Open Sport Exerc Med 2016;2:e000043.

22 Fuller CW, Molloy MG, Bagate C, et al. Consensus statement on injury definitions and data collection procedures for studies of injuries in rugby union. Clin I Sport Med 2007;17:177-81.

23 Rae K, Orchard J. The Orchard Sports Injury Classification System (OSICS) version 10. Clin I Sport Med 2007:17:201-4.

24 Hopkins WG. Generalized linear mixed models in SPSS. SportSci 2016;20.

25 Herman K, Barton C, Malliaras P, et al. The effectiveness of neuromuscular warm-up strategies, that require no additional equipment, for preventing lower limb injuries during sports participation: a systematic review. BMC Med 2012;10:75.
26 Hopkins WG. A spreadsheet for deriving a confidence inteval, mechanistic inference and confidence inference from a p-value. Sportsci 2007:11.

27 Hopkins WG. Linear models and effect magnitudes for research, clinical and practical applications. SportSci 2010;14.

28 Batterham AM, Hopkins WG. Making meaningful inferences about magnitudes. Int $\mathrm{J}$ Sports Physiol Perform 2006;1:50-7.

29 Batten J, White AJ, Anderson E, et al. From management to prevention: the new cure for sports concussion. Br J Sports Med 2016:50:1293-4.

30 Benson BW, McIntosh AS, Maddocks D, et al. What are the most effective riskreduction strategies in sport concussion? Br J Sports Med 2013;47:321-6.

31 Hamilton DF, Gatherer D, Robson J, et al. Comparative cervical profiles of adult and under-18 front-row rugby players: implications for playing policy. BMJ Open 2014;4:e004975.

32 Patel DR, Shivdasani V, Baker RJ. Management of sport-related concussion in young athletes. Sports Med 2005:35:671-84.

33 Schneider KJ, Meeuwisse WH, Kang J, et al. Preseason reports of neck pain, dizziness, and headache as risk factors for concussion in male youth ice hockey players. Clin J Sport Med 2013;23:267-72

34 Schneider KJ, Emery CA, Kang J, et al. Examining sport concussion assessment tool ratings for male and female youth hockey players with and without a history of concussion. Br J Sports Med 2010;44:1112-7.

35 Shehata N, Wiley JP, Richea S, et al. Sport concussion assessment tool: baseline values for varsity collision sport athletes. Br I Sports Med 2009;43:730-4.

36 Lark SD, McCarthy P. The effects of a rugby playing season on cervical range of motion. J Sports Sci 2010;28:649-55.

37 Lark SD, McCarthy PW. Cervical range of motion and proprioception in rugby players versus non-rugby players. J Sports Sci 2007;25:887-94.

38 Lark SD, McCarthy PW. The effects of a single game of rugby on active cervical range of motion. J Sports Sci 2009;27:491-7.

39 Maconi F, Venturelli M, Limonta E, et al. Effects of a 12-week neck muscles training on muscle function and perceived level of muscle soreness in amateur rugby players. Sport Sci Health 2016;12:443-52.

40 Bleakley C, Tully M, O'Connor S. Epidemiology of adolescent rugby injuries: a systematic review. J Athl Train 2011;46:555-65.

41 Steffen K, Andersen TE, Krosshaug T, et al. ECSS position statement 2009: prevention of acute sports injuries. Eur I Sport Sci 2010;10:223-36.

42 Ogaki R, Takemura M, Iwai K, et al. Risk factors for shoulder injury in collegiate Rugby union players. Inter I Sport Health Sci 2014;12:31-7.

43 Andersson SH, Bahr R, Clarsen B, et al. Preventing overuse shoulder injuries among throwing athletes: a cluster-randomised controlled trial in 660 elite handball players. Br J Sports Med 2016

44 Niederbracht Y, Shim AL, Sloniger MA, et al. Effects of a shoulder injury prevention strength training program on eccentric external rotator muscle strength and glenohumeral joint imbalance in female overhead activity athletes. I Strength Cond Res 2008:22:140-5.

45 Soligard T, Nilstad A, Steffen K, et al. Compliance with a comprehensive warm-up programme to prevent injuries in youth football. Br J Sports Med 2010:44:787-93.

46 Hägglund M, Atroshi I, Wagner P, et al. Superior compliance with a neuromuscular training programme is associated with fewer $\mathrm{ACL}$ injuries and fewer acute knee injuries in female adolescent football players: secondary analysis of an RCT. Br I Sports Med 2013;47:974-9.

47 Impellizzeri FM, Bizzini M, Dvorak J, et al. Physiological and performance responses to the FIFA 11+ (part 2): a randomised controlled trial on the training effects. J Sports Sc 2013:31:1491-502.

48 Fortington LV, Donaldson A, Lathlean T, et al. When 'just doing it' is not enough: assessing the fidelity of player performance of an injury prevention exercise program. J Sci Med Sport 2015;18:272-7.

49 Hanson D, Allegrante JP, Sleet DA, et al. Research alone is not sufficient to prevent sports injury. Br J Sports Med 2014;48:682-4.

50 Twomey D, Finch C, Roediger E, et al. Preventing lower limb injuries: is the latest evidence being translated into the football field? I Sci Med Sport 2009;12:452-6. 